\title{
SOME MAXIMAL TOPOLOGIES WHICH ARE QHC
}

\author{
DOUGLAS E. CAMERON
}

\begin{abstract}
In this paper we characterize maximal $C$-compact spaces, maximal QHC spaces, and maximal nearly compact spaces. We also discuss a covering property which turns out to be equivalent to $S$-closed and extremally disconnected.
\end{abstract}

1. C-compact spaces. In 1969 Giovanni Viglino [V1] introduced the concept of $C$-compact spaces and showed that a Hausdorff $C$-compact space is minimal Hausdorff (and thus maximal $C$-compact). In this first section we shall characterize all maximal $C$-compact spaces. The author wishes to thank the referee whose suggestions improved the exposition of this paper.

The concept of a $C$-compact space utilizes the concept of QHC-subspaces. A topological space is quasi- $\boldsymbol{H}$-closed $(\mathrm{QHC})$ if every open cover has a finite proximate subcover; that is, every open cover has a finite subfamily whose union is dense in the space or equivalently a finite subfamily whose closures cover the space. A subset $A$ of a topological space $(X, \tau)$ is $\mathrm{QHC}$ if it is $\mathrm{QHC}$ in its relative topology. A subset $A$ of a topological space $(X, \tau)$ is $\mathrm{QHC}$ relative to $\tau$ if every $\tau$-open cover of $A$ has a finite subfamily whose $\tau$-closures cover the set. A QHC subset is QHC relative to $\tau$ but not conversely [PT1]. A space is $C$-compact if every closed subset is $\mathrm{QHC}$ relative to $\tau$.

A topological space $(X, \tau)$ with property $R$ is maximal $R$ [minimal $R$ ] if whenever $\tau^{*} \supset \tau\left[\tau^{*} \subset \tau\right],\left(X, \tau^{*}\right)$ does not have property $R$.

The following was proved by Viglino [V1]: In Hausdorff spaces, compact $\rightarrow C$-compact $\rightarrow$ minimal $T_{2}$, and the implications are not reversible.

From its very definition it is obvious that every $C$-compact space is QHC. Maximal QHC spaces have been studied by the author in [C2] and included in a general class of spaces in [C3]. $C$-compactness is not of the latter class since the properties included in this class are contractive, contagious, preserved by finite unions, semiregular, and regular closed hereditary. $C$-compactness is contractive [S1] and preserved by finite unions (Theorem 1), but is not contagious (Example 1), semiregular (Example 2), or regular closed hereditary [S1].

A property is contagious if whenever it is possessed by a dense subset of a space then the entire space has the property. Examples of contagious properties are connectedness, separability, and QHC.

Received by the editors June 21, 1978 and, in revised form, September 6, 1978.

AMS (MOS) subject classifications (1970). Primary 54D99.

Key words and phrases. Maximal topologies, $C$-compact, QHC, nearly compact, $S$-closed, submaximal. 
EXAMPLE 1. Let $X=[0,1] \cup M$, where $M$ is the set of integers greater than 1 . [0, 1] with the usual topology is a dense, open subspace of $X$, and if $n \in M$, a neighborhood of $n$ is $\{n\}$ together with a deleted neighborhood of $1 / n$ in $[0,1]$. $[0,1]$, being compact, is $C$-compact, and $M$ is closed in $X$. However, $M$ is not QHC relative to $\tau$, as may be seen by considering the $\tau$-open cover $\left\{\{n\} \cup\left(1 / n-r_{n}, 1 / n+r_{n}\right): n \in M\right\}$ of $M$, where $r_{n}=\frac{1}{2}(1 / n-1 /(n+$ 1)).

THEOREM 1. C-compactness is preserved by finite unions.

Proof. Let $X=A \cup B$ where $(A, \tau \mid A)$ and $(B, \tau \mid B)$ are $C$-compact. If $K$ is a closed subset of $X$ and $\left\{U_{\alpha} \mid \alpha \in \Delta\right\}$ is a $\tau$-open cover of $K$ then $\left\{U_{\alpha} \cap A \mid \alpha\right.$ $\in \Delta\}$ is a $\tau \mid A$ cover of $K \cap A$ (similarly for $B$ ). Thus there is a $\tau \mid A$ finite proximate subcover of $K \cap A$ and similarly for $B$. The union of these two proximate subcovers is a proximate subcover of $K$. Q.E.D.

A topological property $R$ is semiregular if the space $(X, \tau)$ has property $R$ whenever $\left(X, \tau_{S}\right)$ has property $R$ where $\tau_{S}$ is the semiregularization of $\tau$, or the topology on $X$ which has as its base the regular open sets of $\tau$. A topology $\tau$ is semiregular if $\tau=\tau_{s}$.

For a topological space $(X, \tau)$ and a subset $A \subset X$ such that $A \notin \tau$, the topology $\tau(A)=\{U \cup(V \cap A) \mid U, V \in \tau\}$ is called the simple expansion of $\tau$ by $A$. $(\tau(A)$ is just the topology generated by $\tau \cup\{A\}$ as a subbase.)

EXAMPLE 2 . The interval $[0,1]$ with the usual topology $\tau$ is $C$-compact since it is compact and, being Hausdorff, it is minimal Hausdorff and thus maximal $C$-compact. However, if $Q$ is the set of rational numbers in $[0,1]$, then $\tau(Q)$ has $\tau$ as its semiregularization (minimal Hausdorff spaces are semiregular), but $\tau(Q)$ is not $C$-compact.

Though $C$-compactness is neither closed hereditary [V1] nor regular closed hereditary [S1], the property is preserved somewhat as the following shows.

THEOREM 2. If $B$ is a regular closed subset of a $C$-compact space $(X, \tau)$, then $(B, \tau \mid B)$ is $Q H C$.

Proof. This follows from the fact that $C$-compact spaces are QHC and the fact that a space is QHC if and only if every regular closed subset is QHC [C2]. Q.E.D.

The preceding does not hold for closed subsets (the closed subset $\{(1 / n, 0) \mid n \in Z\}$ of Example 2 in [V1] is a counterexample) although closed subsets are interiorly QHC [C2]. A subset $A$ of a topological space $(X, \tau)$ is interiorly $\mathrm{QHC}$ if every open cover of $A$ has a finite subfamily whose closures cover int $\boldsymbol{\tau}$.

We shall now proceed to an examination of the characterization of maximal $C$-compact spaces. To facilitate this investigation we want to introduce two new concepts.

Definition 1. A subset $A$ of a topological space $(X, \tau)$ is $C$-compact relative to $\tau$ if every relative closed subset of $A$ is QHC relative to $\tau$. 
The following is obvious.

TheOREM 3. $(X, \tau)$ is $C$-compact if and only if every proper closed set is $C$-compact relative to $\tau$.

THEOREM 4. $C$-compactness relative to $\tau$ is contractive.

Proof. If $\tau^{*} \subset \tau$, then every $\tau^{*}$ cover of a relative closed subset of the subspace $\left(A, \tau^{*} \mid A\right)$ is a $\tau$ cover and the result follows. Q.E.D.

THEOREM 5. C-compactness relative to $\tau$ is relative closed hereditary.

Proof. If $(X, \tau \mid A)$ is $C$-compact relative to $\tau$, and $K_{2}$ is a relative closed subset of the relative closed subset $K \cap A$, where $K$ is closed, then $K_{2}=K_{1}$ $\cap(K \cap A)=\left(K_{1} \cap K\right) \cap A$ for some closed $K_{1}$ and is therefore a $\tau \mid A$ closed subset of $A$. Q.E.D.

$[0,1]$ is $C$-compact relative to the usual topology $\tau$ but is not $C$-compact relative to $\tau(Q)$ where $Q$ is the set of rationals in $[0,1]$. Thus this property is not semiregular.

Example 1 shows that $C$-compactness relative to $\tau$ is not contagious. The proof of the following is easily obtained.

TheOREM 6. If $(X, \tau)$ is Hausdorff and $A$ is $Q H C$ relative to $\tau$, then $A$ is closed. In particular, if $(X, \tau)$ is Hausdorff and $A$ is $C$-compact relative to $\tau$, then $A$ is $\tau$-closed.

Definition 2. A subset $A$ of $(X, \tau)$ is closure $C$-compact if every $\tau$-open cover of $\operatorname{cl}_{\tau} K$ has a $\tau \mid A$ finite proximate subcover of $K$, where $K$ is a $\tau \mid A$ closed subset of $A$.

Since closed subsets of $C$-compact spaces are not necessarily QHC, it follows that $C$-compactness relative to $\tau$ does not imply closure $C$-compactness. Also, closure $C$-compactness does not imply $C$-compactness relative to $\tau$. ]0, 1[ is closure $C$-compact as a subspace of $[0,1]$ with the usual topology but is not $C$-compact relative to the usual topology since it is not closed.

THeOREM 7. Closure C-compactness is contractive.

Proof. Let $A$ be closure $C$-compact in $(X, \tau), \tau^{*} \subset \tau$, and let $K$ be a $\left(\tau^{*} \mid A\right)$-closed subset of $A$. Then $K$ is $(\tau \mid A)$-closed, and a $\tau^{*}$-open cover of $\mathrm{cl}_{\tau^{*}} K$ is a $\tau$-open cover of $\mathrm{cl}_{\tau} K \subseteq \mathrm{cl}_{\tau^{*}} K$. Thus, such a cover of $\mathrm{cl}_{\tau^{*}} K$ has a finite, proximate $\tau \mid A$ subcover of $K$. Q.E.D.

TheOREM 8. A space $(X, \tau)$ is $C$-compact if and only if every open set is closure C-compact.

Proof. Let $(X, \tau)$ be $C$-compact, $T$ open and $K \subseteq T$ be $\tau \mid T$ closed. If $\left\{U_{\alpha} \mid \alpha \in \Delta\right\}$ is an open cover of $\mathrm{cl}_{\tau} K$, then there is a $\tau$ finite proximate subcover of $\mathrm{cl}_{\tau} K$. Since $\mathrm{cl}_{\tau \mid T}\left(U_{\alpha} \cap T\right)=\left(\mathrm{cl}_{\tau} U_{\alpha}\right) \cap T, T$ is closure $C$ compact. 
If all open sets are closure $C$-compact, let $\mathscr{U}=\left\{T_{\alpha} \mid \alpha \in \Delta\right\}$ be a $\tau$-open cover of a closed set $K$. Then select $T_{\alpha_{1}} \in \mathscr{Q} . T^{*}=X-\operatorname{cl}_{\tau} T_{\alpha_{1}}$ is open and $K \cap T^{*}$ is relative closed in $T^{*}$ with $\left\{T_{\alpha} \mid \alpha \in \Delta-\left\{\alpha_{1}\right\}\right\}$ as a $\tau$-cover. Then there is a $\tau \mid T^{*}$ finite proximate subcover of $K \cap T^{*}$, and this, together with $T_{\alpha_{1}}$, is a $\tau$ finite proximate cover of $K$. Q.E.D.

Thus since $C$-compactness is not productive neither is closure $C$-compactness.

TheOREM 9. $(X, \tau)$ is maximal $C$-compact if and only if whenever $A \subseteq X$ is such that $A$ is closure $C$-compact and $X-A$ is $C$-compact relative to $\tau$, then $A$ is open.

Proof. If $(X, \tau)$ is maximal $C$-compact and $A$ satisfies the conditions, let $K$ be $\tau(A)$-closed. Then $K=K_{1} \cup\left(K_{2} \cap(X-A)\right)$ where $K_{1}, K_{2}$ are $\tau$-closed. Then for any $\tau(A)$-open cover $\left\{U_{\alpha} \cup\left(V_{\alpha} \cap A\right) \mid U_{\alpha}, V_{\alpha} \in \tau, \alpha \in \Delta\right\}$ of $K,\left\{U_{\alpha} \mid \alpha \in \Delta\right\}$ is a $\tau$ cover of $K \cap(X-A)=\left(K_{1} \cup K_{2}\right) \cap(X-A)$. Since $X-A$ is $C$-compact relative to $\tau,\left\{U_{\alpha} \mid \alpha \in \Delta\right\}$ has a finite proximate $\tau$ (and hence $\tau(A)$ ) subcover of $K \cap(X-A)$. $\left\{U_{\alpha} \cup V_{\alpha} \mid \alpha \in \Delta\right\}$ is a $\tau$ cover of $\mathrm{cl}_{\tau}(K \cap A)=\operatorname{cl}_{\tau}\left(K_{1} \cap A\right)=\mathrm{cl}_{\tau(A)}\left(K_{1} \cap A\right)$, and thus there is a $\tau \mid A=$ $\tau(A) \mid A$ finite proximate subcover of the cover. These two subcovers together form a proximate $\tau(A)$ cover of $K$. Thus $\tau(A)$ is $C$-compact and since $\tau$ is maximal $C$-compact, $\tau=\tau(A)$ and $A \in \tau$.

If $(X, \tau)$ is not maximal $C$-compact then there is $\tau^{*} \supset \tau$ such that $\left(X, \tau^{*}\right)$ is $C$-compact. For $A \in \tau^{*}-\tau, A$ is $\tau^{*}$ closure $C$-compact by Theorem 8 and thus $\tau$ closure $C$-compact by Theorem 7. Also, $X-A$ is $C$-compact relative to $\tau^{*}$ by Theorem 3 and thus $C$-compact relative to $\tau$ by Theorem 4. Q.E.D.

COROllary 1. A C-compact Hausdorff space is maximal C-compact and therefore minimal Hausdorff.

Proof. This follows from Theorems 3,6 and 9. Q.E.D.

In [C1] we gave an example of a non-Hausdorff QHC space which is maximal $\mathrm{QHC}$, and since it is compact, it is maximal $C$-compact. Also, being maximal QHC it is submaximal [C2], and thus maximal $C$-compact spaces may be submaximal. (A space is submaximal if every dense subset is open.)

In [V1] there is an example of a Hausdorff $C$-compact space which is not compact. $[0,1]$ with the usual topology is maximal $C$-compact but not maximal $\mathrm{QHC}$ although there is a finer topology on $[0,1]$ which is maximal QHC [C2].

2. QHC spaces. In this section we shall extend the concept of closure $C$-compactness to obtain a characterization for maximal $\mathrm{QHC}$ spaces which is similar to that for maximal $C$-compact spaces.

Definition 3. A subset $A$ of a topological space $(X, \tau)$ is closure $Q H C$ if every open cover of $\mathrm{cl}_{\tau} A$ has a finite $\tau \mid A$ proximate cover of $A$. In other 
words, if $\left\{U_{\alpha} \mid \alpha \in \Delta\right\}$ covers $\operatorname{cl}_{\tau} A$, then there exist $U_{\alpha_{1}}, U_{\alpha_{2}}, \ldots, U_{\alpha_{n}}$ such that $\cup_{i=1}^{n}\left(U_{\alpha_{i}} \cap A\right)$ is dense in $A$.

The following examples show that the concepts of closure QHC and interiorly $\mathrm{QHC}$ are distinct.

EXAMPLe 3. $[0,1] \cup \mathbf{N}$, where $\mathbf{N}$ is the set of natural numbers, is interiorly QHC but not closure QHC in the real line.

EXAMPLE 4. In a Hausdorff space $(X, \tau)$, a subset $A$ is interiorly $\mathrm{QHC}$ only if $\mathrm{cl}_{\tau}\left(\right.$ int $\left._{\tau} A\right) \subseteq A$ [C2], which is not true for open sets: in QHC spaces they are closure QHC (see the following theorem).

THEOREM 10. $(X, \tau)$ is $Q H C$ if and only if every open set is closure $Q H C$.

'If' is trivial and 'only if' is much the same as in the proof of Theorem 8.

THEOREM 11. $(X, \tau)$ is maximal $Q H C$ if and only if for $A \subseteq X$ such that $X-A$ is interiorly $Q H C$ and $A$ is closure $Q H C$, then $A$ is open.

Proof. If $(X, \tau)$ is maximal $\mathrm{QHC}$ and $A \subseteq X$ is closure $\mathrm{QHC}$ and $X-A$ is interiorly QHC, let $\left\{U_{\alpha} \cup\left(V_{\alpha} \cap A\right) \mid \alpha \in \Delta\right\}$ be a $\tau(A)$ open cover of $X$. Then $\left\{U_{\alpha} \mid \alpha \in \Delta\right\}$ covers $X-A$ and thus a finite proximate $\tau(A)$ subcover of int $_{\tau}(X-A)=$ int $_{\tau(A)}(X-A)$ exists. $\left\{U_{\alpha} \cup V_{\alpha} \mid \alpha \in \Delta\right\}$ covers $\mathrm{cl}_{\tau} A=\mathrm{cl}_{\tau(A)} A$ and thus there is a finite subfamily whose union is dense in $A$ and thus in $\mathrm{cl}_{\tau} A=X-\left(\operatorname{int}_{\tau}(X-A)\right)$. These two finite subfamilies give us the desired finite proximate cover of $X$. Thus $\tau=\tau(A)$ and so $A \in \tau$.

If $(X, \tau)$ is not maximal QHC then there is $\tau^{*} \supset \tau$ such that $\left(X, \tau^{*}\right)$ is QHC. Then for any $A \in \tau^{*}-\tau, X-A$ is $\tau^{*}$ interiorly QHC and thus $\tau$ interiorly QHC, and $A$ is $\tau^{*}$ closure QHC and thus $\tau$ closure QHC. Q.E.D.

This theorem improves upon the characterizations for maximal QHC spaces in [C2] in that the proofs there relied heavily upon the fact that the maximal QHC spaces are submaximal. The concept of closure QHC helps us obtain this fact now in a different manner.

THEOREM 12. If $(X, \tau)$ is $Q H C$ and $A$ is a dense subset, then $A$ is closure $Q H C$.

Proof. If $\left\{U_{\alpha} \mid \alpha \in \Delta\right\}$ is a $\tau$ open cover of $\operatorname{cl}_{\tau} A=X$, then there is a finite proximate subcover $\left\{U_{\alpha_{i}} \mid i=1,2, \ldots, n\right\}$ of $X$. If $x \in A, x \in T \in \tau$, and $T \cap U_{\alpha_{i}} \neq \varnothing$, then $T \cap U_{\alpha_{i}} \cap A \neq \varnothing$ since $A$ is dense, and thus $\left\{U_{\alpha_{i}} \cap A \mid i\right.$ $=1,2, \ldots, n\}$ is a finite $\tau \mid A$ proximate subcover of $A$. Q.E.D.

COROLlARY 2. Maximal QHC spaces are submaximal.

Proof. If $A$ is dense then $X-A$ is interiorly QHC since int $\tau(X-A)=\phi$, and the result follows from Theorems 11 and 12 and the fact that a space is submaximal if and only if the dense subsets are open. Q.E.D.

3. I-compact spaces. The $S$-closed spaces introduced by Travis Thompson [T1] have been characterized as those spaces in which every cover by regular 
closed sets has a finite subcover [C4]. S-closed spaces are QHC, Hausdorff $S$-closed spaces are extremally disconnected [T1], and there are $S$-closed spaces which are not extremally disconnected [C4]. Maximal $S$-closed spaces are precisely the maximal $\mathrm{QHC}$ spaces which are extremally disconnected. A property which is closely related to $S$-closure is that of $I$-compactness.

Definition 4. A topological space $(X, \tau)$ is $I$-compact if every cover by regular closed sets has a finite subfamily whose interiors cover the space.

It is obvious that every $I$-compact space is $S$-closed and that an extremally disconnected $S$-closed space is $I$-compact. We shall show that the $I$-compact spaces are precisely the $S$-closed spaces which are extremally disconnected.

LEMMA 1. If $A$ is a regular closed subset of an I-compact space, then every cover of $A$ by $\tau$ regular closed sets has a finite subfamily whose $\tau$ interiors cover A.

Proof. Let $\left\{K_{\alpha} \mid \alpha \in \Delta\right\}$ be a cover of $A$ by $\tau$ regular closed sets. Then $\left\{K_{\alpha} \mid \alpha \in \Delta\right\} \cup\left\{\mathrm{cl}_{\tau}(X-A)\right\}$ is a cover of $X$ by $\tau$ regular closed sets and therefore there is a finite subfamily whose interiors cover $X:\left\{\right.$ int $_{\tau} K_{\alpha_{i}} \mid i=$ $1,2, \ldots, n\} \cup\{X-A\}$ covers $X$. Thus $\left\{\right.$ int $\left._{\tau} K_{\alpha_{i}} \mid i=1,2, \ldots, n\right\}$ covers $A$. Q.E.D.

THEOREM 13. An I-compact space is extremally disconnected.

Proof. Assume that $(X, \tau)$ is $I$-compact and is not extremally disconnected. Then there exist disjoint nonempty open sets $U_{1}$ and $U_{2}$ such that $\mathrm{cl}_{\tau} U_{1} \cap \mathrm{cl}_{\tau} U_{2} \neq \varnothing . \quad A=\mathrm{cl}_{\tau} U_{1} \cup \mathrm{cl}_{\tau} U_{2}$ is regular closed and $\left\{\mathrm{cl}_{\tau} U_{1}, \mathrm{cl}_{\tau} U_{2}\right\}$ is a cover of $A$ by regular closed sets whose interiors do not cover $A$. This contradicts Lemma 1. Q.E.D.

Corollary 3. A space is I-compact if and only if it is extremally disconnected and $S$-closed.

4. Nearly compact spaces. The nearly compact spaces introduced by $M$. K. Singal and A. Mathur [SM1] are characterized as those spaces in which every cover by regular open sets has a finite subcover. Since QHC spaces may be characterized as those spaces in which every cover by regular open sets has a finite proximate subcover, we have the following implications, none of which is reversible: compact $\rightarrow$ nearly compact $\rightarrow \mathrm{QHC}$.

THEOREM 14. $(X, \tau)$ is nearly compact if and only if $\left(X, \tau_{S}\right)$ is compact [Ca1].

Theorem 14 shows that nearly compact spaces are semiregular and it is known that near compactness is regular closed hereditary [SM2]; however, near compactness is not preserved by finite unions [SM2] and thus is not in the class of spaces studied in [C3]. Because near compactness is semiregular, maximal nearly compact spaces are submaximal [C3]. In fact, in view of Theorem 14 and the fact that $\tau_{S}^{*} \neq \tau_{S}$ if $\tau^{*} \supset \tau$ and $\tau$ is submaximal, we have the following: 
THEOREM 15. A nearly compact space $(X, \tau)$ is maximal nearly compact if and only if

(i) $(X, \tau)$ is submaximal and not compact, or

(ii) $(X, \tau)$ is submaximal and maximal compact.

A topological space $(X, \tau)$ with property $R$ is strongly $R$ if there is a finer topology $\tau^{*}$ such that $\left(X, \tau^{*}\right)$ is maximal $R$.

COROLlary 4. A maximal compact space is strongly nearly compact.

COROLlARY 5. A nearly compact space which is not compact is strongly nearly compact.

These results follow from the fact that every topological space has a finer submaximal topology which is ro-equivalent [B1]. (Two topologies are roequivalent if they have the same semiregularization.)

The example discussed in [C1] is a maximal compact space which is submaximal and therefore maximal nearly compact. It is also maximal QHC and not Hausdorff.

$[0,1]$ with the usual topology is maximal compact but it not submaximal and therefore not maximal nearly compact.

Example 6 of [C2] is a maximal QHC space which is not nearly compact since it is not completely Hausdorff. (A Hausdorff space is nearly compact if and only if it is QHC and completely Hausdorff [H1].)

N. Smythe and C. A. Wilkins [SW1] constructed a maximal compact space which is not submaximal but is, of course, strongly nearly compact. They also have a minimal Hausdorff space which is finer than their maximal compact space. Since minimal Hausdorff spaces are semiregular, this space is not ro-equivalent to the maximal compact space, and since minimal Hausdorff spaces are strongly QHC there is a maximal nearly compact space which is not maximal QHC.

\section{REFERENCES}

[B1] N. Bourbaki, Elements of mathematics. General topology, Parts I, II, Addison-Wesley, Reading, Mass., 1966.

[Ca1] D. Camahan, On locally nearly-compact spaces, Boll. Un. Mat. Ital. 6 (1972), 146-153.

[C1] Douglas E. Cameron, Hausdorff and H-compact are not complementary, Rocky Mountain J. Math. 7 (1977), 123-124.

[C2] Maximal QHC spaces, Rocky Mountain J. Math. 7 (1977), 313-322.

[C3] _ A class of maximal topologies, Pacific J. Math. 70 (1977), 101-104.

[C4] _ Properties of S-closed spaces, Proc. Amer. Math. Soc. 72 (1978), 581-586.

[H1] Larry L. Herrington, Properties of nearly-compact spaces, Proc. Amer. Math. Soc. 45 (1974), 431-436.

[HL1] Larry L. Herrington and Paul E. Long, Characterizations of C-compact spaces, Proc. Amer. Math. Soc. 52 (1975), 417-426.

[J1] James E. Joseph, Characterizations of nearly-compact spaces, Boll. Un. Mat. Ital. 13 (1976), 311-321.

[J2] , Characterizations of nearly-compact spaces. II, Boll. Un. Mat. Ital. 13 (1976), $601-606$. 
[PT1] Jack Porter and John Thomas, On H-closed and minimal Hausdorff spaces, Trans. Amer. Math. Soc. 138 (1961), 159-170.

[S1] Shozo Sakai, A note on C-compact spaces, Proc. Japan Acad. 46 (1970), 917-920.

[SM1] M. K. Singal and Asha Mathur, On nearly compact spaces, Boll. Un. Math. Ital. (4) 2 (1969), 702-710.

[SM2] __, On nearly compact spaces. II, Boll. Un. Math. Ital. 9 (1974), 670-678.

[SW1] N. Smythe and C. A. Wilkins, Minimal Hausdorff and maximal compact spaces, J. Austral. Math. Soc. 3 (1963), 167-171.

[T1] Travis Thompson, S-clased spaces, Proc. Amer. Math. Soc. 60 (1976), 335-338.

[T2] _ Characterizations of nearly compact spaces, Kyungpook Math. J. 17 (1977), 37-41.

[V1] Giovanni Viglino, C-compact spaces, Duke Math. J. 36 (1969), 761-764.

Department of MAthematics, UNiversity of AKron, AKron, OHo 44325 